\title{
Exteroception and exproprioception by dynamic touch are different functions of the inertia tensor
}

\author{
CHRISTOPHER C. PAGANO \\ Center for the Ecological Study of Perception and Action \\ University of Connecticut, Storrs, Connecticut \\ and Clemson University, Clemson, South Carolina \\ and \\ CLAUDIA CARELLO and M. T. TURVEY \\ Center for the Ecological Study of Perception and Action \\ University of Connecticut, Storrs, Connecticut \\ and Haskins Laboratories, New Haven, Connecticut
}

\begin{abstract}
When an object is held and wielded, a time-invariant quantity of the wielding dynamics is the inertia tensor $I_{i j}$. The $3 \times 3$ quantity $I_{i j}$ is composed of moments of inertia (on the diagonal) and products of inertia (off the diagonal). Examination of $I_{i j}$ as a function of different locations at which a cylindrical object is grasped revealed that the products related systematically to grip position (a direction), and both the products and moments taken together related systematically to the extent of the rod to one side of the hand (a magnitude in a direction). In two experiments, observers wielded an occluded rod that was held at an intermediate point along its length and reproduced both the felt grip position and partial rod length. In both experiments, perceived grip position was a function of the rod's products of inertia and perceived partial rod length was a function of the moments and products. Discussion focuses on the specificity of exteroception and exproprioception to $I_{i j}$.
\end{abstract}

When one firmly grasps and manipulates an occluded object, there is conjointly perception of properties of the object and how the body segments and the object are oriented relative to each other. For example, when an object such as a ruler, a hammer, a hockey stick, or a cane is held at a position part way between its two ends, one seems to have nonvisual impressions of the overall magnitude of the object, the magnitude of the portion of the object that lies to one side of the hand, the position of the hand along the object, and the orientation of the object relative to the hand. These concurrent haptic perceptions of holding must play a significant role in the control of actions involving the held object. In the present article, we address the potential basis for the haptic ability to perceive the related properties of grasp position along a wielded object and the magnitude of the portion of the object that lies to one side of the hand. Following Sherrington (1906), perception of the latter kind, directed at an object property, is commonly called exteroception. Perception of the for-

This work was supported by National Science Foundation Grant SBR 93-09371, awarded to M.T.T. and C.C., and by U.S. Public Health Service Individual National Research Service Award IFS32NS0957501 to C.C.P. Preliminary results of Experiment 1 were presented at the North American Meeting of the International Society for Ecological Psychology, Northampton, MA, October 1993. The authors would like to thank Nileshkumar Sutaria for running Experiment 2, and Geoffrey Bingham for helpful discussions pertaining to this work. Correspondence should be addressed to C. C. Pagano, Department of Psychology, 418 Brackett Hall, Box 341511, Clemson University, Clemson, SC 29634-1511 (e-mail: cpagano@clemson.edu). mer kind, directed at the hand's orientation to an object, is uncommonly called exproprioception following Lee (1978). Whereas the classical term proprioception refers to the perception of orientations of segments of the body to each other and to the body as a whole, the newly coined term exproprioception refers to the perception of orientations of body segments, and of the body as a unit, to the objects and events of the environment. In the present article, therefore, we address the joint capabilities of haptic exteroception and exproprioception.

The haptic subsystem of most importance in the preceding examples is dynamic touch. It relates primarily to the tensile states of muscles and tendons as these tissues undergo deformation during exploratory and performatory activity (Gibson, 1966). Gibson found it useful to differentiate among three types of touch-specifically, cutaneous, haptic, and dynamic - each of which can be considered as a subsystem of the haptic perceptual system. Cutaneous touch is the stimulation of skin and deeper tissues without movements of joints. Haptic touch is the stimulation of the skin and deeper tissues with movements of the joints. Dynamic touch involves the stimulation of skin and other tissues in combination with muscular exertion. Gibson explained that dynamic touch "is a perceptual system in its own right. More than any others, it is perception blended with performance, for the information comes from muscular effort" (p. 128). In some important respects, however, Gibson's distinction between haptic and dynamic touch has not stood the test of time. According to his definitions, haptic touch involves the 
pickup of spatial information from cutaneous and joint mechanoreceptors, whereas dynamic touch involves the combination of information from cutaneous and joint mechanoreceptors with nonspatial information from the muscles. It is clear that his definition of haptic touch involves the joint sense and not the muscle sense: "The evidence strongly suggests that muscle sensitivity is irrelevant for the perception of space and movement, whereas joint sensitivity is very important to it. In short, we detect the angles of our joints" (p. 109). We now know, however, that the muscle sense, and not the joint sense, is responsible for the spatial information being referred to in Gibson's definition of haptic touch. That is, research conducted since the time Gibson wrote has revealed that the muscle sense, and not the joint sense, is primarily responsible for the perception of limb position and movement (e.g., Burgess, Wei, Clark, \& Simon, 1982; Clark \& Horch, 1986; Kelso, 1978; Matthews, 1982; McCloskey, 1978; Pagano \& Turvey, 1995). Thus what Gibson termed haptic touch requires the muscle sense, which he termed dynamic touch. Although his definition of dynamic touch is still appropriate, the manner in which he distinguished it from other forms of touch is in need of some revision.

A more recent distinction between the different kinds of touch has been provided by Loomis and Lederman (1986). Within their framework, tactile perception refers to perception based on information arising solely from cutaneous stimulation. Kinesthetic perception refers to the awareness of body posture, wielded objects, and probed surfaces, on the basis of afferent information originating in the muscles, joints, and skin. This includes cases where "cutaneous stimulation serves only to indicate contact with the stimulus, while variations in kinesthetic stimulation convey all of the spatial information essential to the performance of the task" (p. 31-3). Finally, haptic perception refers to cases where both the cutaneous and kinesthetic senses convey information about distal objects and events. The kind of touch classified by Loomis and Lederman as kinesthetic is an apt description of that which is presently investigated under the term dynamic touch (see Turvey \& Carello, 1995, for a review of the dynamic touch literature). However, since the traditional usage (and most common present-day usage) of the term kinesthesis is only with respect to the perception of the body and limbs, the term dynamic touch is often more useful. ${ }^{1}$ The term dynamic touch is more general, referring to use of the muscle sense in the detection of spatial properties of objects and adjacent surfaces. Thus in addition to the perception of the body and limbs, dynamic touch is implied whenever an object is grasped and wielded, whenever a solid surface is palpated or vibrated, and whenever a hand-held implement is brought into contact with other objects (Turvey \& Carello, 1995).

Movements of hand-held objects and body segments relative to each other are always rotational--occurring about one or more joints. Thus the tissue deformations in question are the physical consequences of rotational dy- namics. Rotational motions about a fixed point of the kind characteristic of wielding about a joint follow from

$$
N_{i}=\left(I_{i j} \cdot \dot{\omega}_{j}\right)+\omega_{j} \times\left(I_{i j} \cdot \omega_{j}\right),
$$

where $\cdot$ is the matrix product and $X$ is the vector cross product (Goldstein, 1980). In wielding a given object, the torque $N_{i}$, angular velocity $\omega_{j}$, and angular acceleration $\dot{\omega}_{j}$ vectors are coupled by the inertia tensor $I_{i j}$. Thus $I_{i j}$ is a parameter (a constant) that couples the varying torques and varying rotations of wielding. $I_{i j}$ is represented mathematically by a matrix of numbers. The calculations of these components are done with respect to a rectangular coordinate system $O x y z$. Patently, there are indefinitely many sets of three perpendicular axes $x y z$ that can be anchored at the point of rotation $O$. For each choice of $O x y z$, the components of $I_{i j}$ will differ, but the way in which the tensor specifies properties of the object does not change. This is a basic property of tensors (Lovett, 1989); inertia measured about one set of axes can be transformed to inertia measured about a different set of axes. In general a tensor is a hypernumber - a matrix of numbers that taken together express a physical state of affairs and that transforms in a particularly simple way (Moon \& Spencer, 1986). Different coordinate systems $O x y z$ result in different tensorial components (the numbers in the matrix change), but the manner in which the tensor transforms is such that the tensor as a whole (with all the components considered together) continues to define the object property it quantifies. As a time-independent and coordinateindependent quantity, $I_{i j}$ is an invariant rendering of the persistent material distribution of the hand-held wielded object. With a given point of rotation, $I_{i j}$ does not change; it is a constant property of the rigid object. It can, therefore, be used to quantify the information for perceiving the object's unchanging dimensions (see, e.g., Solomon, 1988; Solomon \& Turvey, 1988; Turvey, 1994). Even when an object's motions occur about several joints-such as the wrist, elbow, and shoulder taken singly or in combination - an invariant rendering of $I_{i j}$ can be found that maps onto perceived object properties (Pagano, Fitzpatrick, \& Turvey, 1993). The implication is that dynamic touch is tuned to the invariant parameters of the object's dynamics, rather than to the varying states (displacements, velocities) and torques (see Amazeen \& Turvey, 1996).

Represented mathematically by a symmetric $3 \times 3$ matrix (Goldstein, 1980), $I_{i j}$ 's diagonal components $\left(I_{x x}\right.$, $I_{y y}, I_{z z}$-referred to as moments of inertia-quantify the object's rotational inertia with respect to the three orthogonal axes of rotation (such as those depicted in Figure 1). $I_{i j}$ 's off-diagonal components $\left(I_{x y}, I_{x z}, I_{y x}, I_{y z}, I_{z x}\right.$, $\left.I_{z y}\right)$-referred to as products of inertia - quantify the object's rotational inertia in directions perpendicular to the axial rotations and reflect the asymmetrical mass distribution of the object about the axes. $I_{y z}$, for example, is the moment about an axis perpendicular to the $y z$ plane of the centrifugal forces caused by rotation about either the 

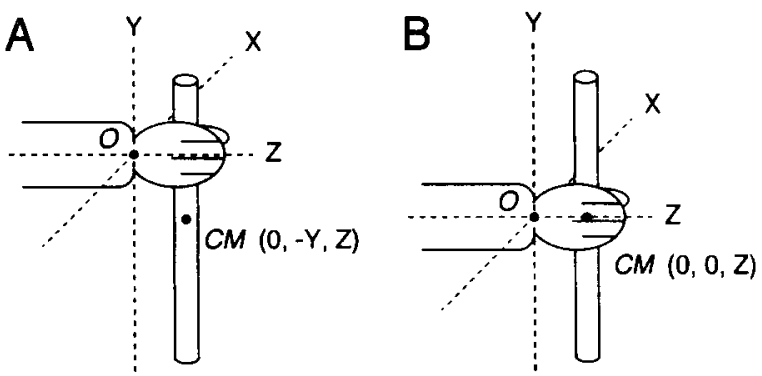

Figure 1. A rod wielded about a fixed point $O$, located in the wrist, with respect to a rectangular coordinate system centered at $O$. The rod is grasped with .25 and .50 of its total length lying above the hand ( $A$ and $B$, respectively).

$y$ - or the $z$-axis (see, e.g., den Hartog, 1948/1961). $I_{i j}$ is a symmetric tensor; accordingly, $I_{i j}=I_{j i}$ and the nine components reduce to six. Recent research on dynamic touch has revealed that the moments of inertia map onto perceived object "magnitudes," such as object length (Fitzpatrick, Carello, \& Turvey, 1994; Pagano et al., 1993; Pagano \& Turvey, 1993; Solomon \& Turvey, 1988; Solomon, Turvey, \& Burton, 1989a, 1989b), shape (Burton, Turvey, \& Solomon, 1990), and weight (Amazeen \& Turvey, 1996). Importantly, this work has investigated other mechanical parameters that are possibly relevant to wielding, such as torque and kinetic energy (and thus angular velocity and acceleration), muscular torsion, mass, center of mass, and center of oscillation, and has ruled out each in favor of $I_{i j}$ (see Amazeen \& Turvey, 1996; Burton \& Turvey, 1990; Solomon \& Turvey, 1988; Solomon et al., 1989a, 1989b). ${ }^{2}$

In some of these experiments, observers were asked to perceive only the length of a hand-held rod that extended to one side of the hand's position of grasp, where the same rod was grasped at different positions along its length during the course of the experiment (Solomon \& Turvey, 1988; Solomon et al., 1989b; see also Burton \& Turvey, 1991). Thus the object property to be perceived, the extent of a rod segment, varied with variations in grip position. Importantly, it was found that perceived partial length was a function of the magnitude of the object to one side of the hand, and was uninfluenced by the magnitude of the object to the opposite (the to-be-ignored) side of the hand. Further analysis revealed that perceived partial length can be predicted from $I_{x x}$ calculated for that part and was independent of $I_{x x}$ of the whole rod. Considering that the tissue deformation consequences of wielding could only be the result of the entire rod, it was hypothesized that the haptic subsystem of dynamic touch decomposed $I_{x x}$ of the whole rod into the $I_{x x}$ of the rod segment forward of the hand and the $I_{x x}$ of the rod segment backward of the hand (Solomon \& Turvey, 1988). In other words, when the task is to perceive the rod extent forward of the hand, dynamic touch filters the required fragment of the rod through subtraction, $I_{x x}$ whole $-I_{x x}$ back $=I_{x x}$ forward. The problem is that such a decomposition is not physically feasible (see Turvey \& Carello, 1995). Given an $I_{x x}$ whole $=10$ (for example), an indefinitely large number of values would satisfy the decomposition (e.g., 9 and 1 or 3 and 7). Additionally, if $I_{x x}$ back must be used to arrive at $I_{x x}$ forward (for example), how is a value for $I_{x x}$ back achieved?

A consideration of $I_{i j}$ rather than $I_{x x}$ suggests a different hypothesis about the basis for the perception of partial rod length by wielding. The hypothesis investigated in the present article states that such perception is specified by $I_{i j}$ taken in its entirety - with both moments and products of inertia contributing to the observer's impression of partial-rod length (see also Pagano, KinsellaShaw, Cassidy, \& Turvey, 1994, for an application of this hypothesis to the perception of grip position). The significance of this hypothesis is twofold. First, it recognizes explicitly the multicomponent nature of rotational inertia; specifically, that it is quantified by a tensor and is inadequately captured by a single component such as $I_{x x}$ (see, e.g., Goldstein, 1980). Second, it predicts that the treatment of $I_{i j}$ in its entirety dispenses with the need to propose an ability of dynamic touch to decompose $I_{x x}$. Furthermore, the significance of considering $I_{i j}$ in its entirety has been underscored by research on the perception of the orientation of an object to the hand (Pagano \& Turvey, 1992; Turvey, Burton, Pagano, Solomon, \& Runeson, 1992), the position of the hand relative to a wielded object (Pagano et al., 1994), the magnitude of one of two things held in the hand (Turvey, Carello, Fitzpatrick, Pagano, \& Kadar, 1996), and the orientation of the upper limbs (Pagano, Garrett, \& Turvey, 1996; Pagano \& Turvey, 1995)-research that reveals a dependence on both moments and products of inertia. ${ }^{3}$

To show how $I_{i j}$ of an entire hand-held object can support the perception of the magnitude of the part of the object that lies in a particular direction, as well as the perception of grip position, we will consider the concepts of moments and products of inertia for the object depicted in Figure 1. The object is a cylindrical rod that lies in the $y z$ plane, and may be held in the hand anywhere along its length. Two such grasp positions, those corresponding to .25 and .50 of the rod's total length lying above the hand, are depicted in the left and right panels of Figure 1, respectively. Inertial moments and products about any point in space, such as $O$, relate to those about the center of mass $(\mathrm{CM})$ through the equations

$$
I_{x x}=M\left(Y^{2}+Z^{2}\right)+I_{x x}^{*}
$$

and

$$
I_{y z}=-M Y Z+I_{y z}^{*} .
$$

Equation 2 is the parallel axis theorem for moments of inertia; Equation 3 is an equivalent theorem for products of inertia (see, e.g., Kibble, 1985). In Equation $2, I_{x x}^{*}$ is the moment of inertia about the $x$-axis referred to CM as origin, $Y$ and $Z$ are coordinates of $\mathrm{CM}$ in the $O$ coordinate system, and $M$ is the mass. By Equation 2, as $Y$ and $Z$ become larger-that is, CM is displaced further from $O-I_{x x}$ becomes larger; synonymously, $I_{x x}$ is smallest when $C M$ is at its minimal distance from the point of rotation $O$. The equations for $I_{y y}$ and $I_{z z}$ are similar. Unlike 
$I_{x x}$ and $I_{z z}, I_{y y}$ does not vary with changes in grip position. This is because the $x$ - and $z$-coordinates of CM remain unaltered with changes in grip position.

In Equation 3, $I_{y z}^{*}$ is the product of inertia referred to $\mathrm{CM}$ and $Y$ and $Z$ are coordinates of $\mathrm{CM}$ with respect to $O$. If the axes chosen at $\mathrm{CM}$ are the principal axes or axes of symmetry (as is the case with homogeneous cylindrical objects), then $I_{y z}{ }^{*}$ and all other products about CM are zero. But as can be seen from Equation 3, this does not mean that $I_{y z}$ and the other products about $O$ are zero. By Equation 3, as $Y$ and $Z$ become larger-that is, as CM is displaced further from $O-I_{y z}$ becomes larger; synonymously, $I_{y z}$ is 0 when CM is at its minimal distance from $O . I_{y z}$ is also 0 whenever $y$ or $z$ is 0 . The equations for $I_{x y}$ and $I_{x z}$ are similar. In each of the object configurations employed presently, $\mathrm{CM}$ has an $x$-coordinate of zero. Thus $I_{x y}=I_{x z}=0$, and $I_{i j}$ consists of four independent components $\left(I_{x x}, I_{y y}, I_{z z}\right.$, and $\left.I_{y z}\right)$. It is further evident from Equation 3 that the sign of $I_{y z}$ is determined by whether $\mathrm{CM}$ lies in the $+Y$ or $-Y$ direction with respect to $O$. The importance of Equation 3 for the present hypothesis lies in the fact that when a given object is grasped at different positions (that is, different $y$ coordinates of $\mathrm{CM}$ ), the corresponding $I_{i j}$ s differ.

As an example of the above equations, $I_{i j}$ s for four homogeneous rods of lengths $45,60,75$, and $90 \mathrm{~cm}$ are presented in Table 1 with $75 \%, 50 \%, 25 \%$, or $5 \%$ of the total rod length extending above the hand (see also Pagano et al., 1994). Table 1 underscores the fact that the magnitude of all $I_{i j}$ components varies with the overall magnitude of the rod, and that the sign and magnitude of $I_{y z}$ vary with the proportion of the total rod length extending above the hand. A multiple regression of hand position (expressed as a proportion of the whole rod length) on the components of $I_{i j}$ reveals that $I_{y z}$ accounts for $79 \%$ of the variance (of the four independent components of

Table 1

$I_{i j}$ for Four Sample Rod Lengths and Four Sample Grip Positions

\begin{tabular}{|c|c|c|c|c|c|c|}
\hline \multirow{2}{*}{$\begin{array}{c}\text { Whole } \\
\text { Rod } \\
\text { Length }(\mathrm{cm})\end{array}$} & \multirow{2}{*}{$\begin{array}{c}\text { Partial } \\
\text { Rod } \\
\text { Length* }\end{array}$} & \multirow{2}{*}{$\begin{array}{c}\text { Grip } \\
\text { Position } \dagger\end{array}$} & \multicolumn{4}{|c|}{$I_{i j}\left(\mathrm{~g} \cdot \mathrm{cm}^{2} / 1,000\right)$} \\
\hline & & & $I_{x x}$ & $I_{y y}$ & $I_{z z}$ & $I_{y z}$ \\
\hline 45 & 2.25 & .05 & 25.24 & 1.48 & 23.76 & 4.99 \\
\hline 45 & 11.25 & .25 & 13.60 & 1.48 & 12.12 & 2.77 \\
\hline 45 & 22.50 & .50 & 8.41 & 1.48 & 6.93 & 0 \\
\hline 45 & 33.75 & .75 & 13.60 & 1.48 & 12.12 & -2.77 \\
\hline 60 & 3.00 & .05 & 58.29 & 1.98 & 56.32 & 8.87 \\
\hline 60 & 15.00 & .25 & 30.71 & 1.98 & 28.74 & 4.93 \\
\hline 60 & 30.00 & .50 & 18.39 & 1.98 & 16.42 & 0 \\
\hline 60 & 45.00 & .75 & 30.71 & 1.98 & 28.74 & -4.93 \\
\hline 75 & 3.75 & .05 & 112.45 & 2.47 & 109.99 & 13.85 \\
\hline 75 & 18.75 & .25 & 58.58 & 2.47 & 56.12 & 7.70 \\
\hline 75 & 37.50 & .50 & 34.53 & 2.47 & 32.07 & 0 \\
\hline 75 & 56.25 & .75 & 58.58 & 2.47 & 56.12 & -7.70 \\
\hline 90 & 4.50 & .05 & 193.01 & 3.00 & 190.06 & 19.95 \\
\hline 90 & 22.50 & .25 & 99.93 & 3.00 & 96.97 & 11.08 \\
\hline 90 & 45.00 & .50 & 58.37 & 3.00 & 55.42 & 0 \\
\hline 90 & 67.50 & .75 & 99.93 & 3.00 & 96.97 & -11.08 \\
\hline
\end{tabular}

Note $-I_{x y}=I_{x z}=0 . \quad{ }^{*}$ Length of the rod above the hand $(\mathrm{cm}) . \quad{ }^{+}$Partial rod length $(\mathrm{cm}) /$ whole rod length $(\mathrm{cm})$.
$I_{i j}$, only $I_{y z}$ was significant after backwards elimination). In confirmation, $I_{i j}$ 's off-diagonal components have been found to provide the major constraint on the perceiving of where an object is grasped (Pagano et al., 1994). A multiple regression of partial rod length on the components of $I_{i j}$ reveals that $I_{y y}$ and $I_{y z}$ account for $97 \%$ of the variance, with the contribution of $I_{y z}$ dominant (partial $F \mathrm{~s}$ of 90.9 and 318.1 for $I_{y y}$ and $I_{y z}$, respectively) and both $I_{x x}$ and $I_{z z}$ removed by backwards elimination. Multiple regression of partial rod length on $I_{x x}$ and $I_{y z}$ accounts for $90 \%$ of the variance, with $I_{y z}$ dominant (partial $F$ s of 22.9 and 113.65 for $I_{x x}$ and $I_{y z}$, respectively). The implication is that the components of $I_{i j}$ for the whole rod, with both moments and products taken together, should support the perception of the magnitude of the part of the object that lies in a particular direction, with $I_{y z}$ providing the major constraint. ${ }^{4}$

\section{EXPERIMENT 1}

If dynamic touch can be attuned selectively to different aspects of the structured array of different resistances to rotation in different directions, $I_{i j}$ for a hand-held cylindrical object ought to be able to support the perception of both partial rod length and grip position. Analysis of $I_{i j}$ reveals systematic covariation (1) between partial length and moments and products of inertia taken together, and (2) between hand position and products of inertia. This selectivity hypothesis was investigated in an experiment in which the observers, on a given trial, wielded one of three wooden rods, each of $60-\mathrm{cm}$ length. One rod had a single 50-g metal ring affixed to one end below the hand, one had two $50 \mathrm{~g}$ metal rings affixed to its ends above and below the hand, and one rod had no metal rings affixed (Figure 2). On a trial, the observer attempted to perceive the position of grasp along the rod, or the length of the portion of the rod that extended above the hand. It was expected that each perception would be a function of $I_{i j}$ for the entire object, with both moments and products of inertia relevant to partial length perception, and with only products of inertia relevant to grip perception.

The expected outcome of the experiment is presented schematically in Figure 3. Grip position should be perceived to be similar in both the no-added-mass and twoadded-mass conditions. This is because within each of these conditions, the mass of the object is evenly distributed to both sides of the hand when the hand is positioned at .50 of the total rod length, as depicted in Figure 2 (note that the mean $I_{y z}$ value is 0 for each of these conditions). In the one-added-mass condition, however, the grip should be perceived to be closer to the top of the object (with less of the object extending above the hand) because there is always a smaller proportion of the object's mass lying above the hand (i.e., there is a mean value of $I_{y z}$ greater than 0 in that condition; see also Pagano et al., 1994). Thus, perceived grip position as a function of the added-mass condition should appear similar to the function depicted in Figure 3A. For perceived 

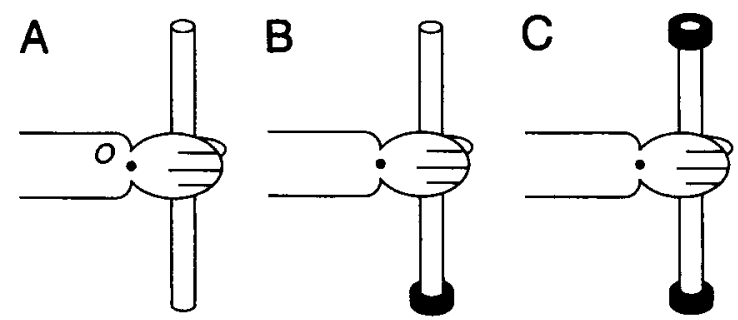

Figure 2. The objects used in the experiments consisted of wooden rods with three different added mass conditions; no mass added to the rod (A), a single 50-g mass affixed to the end of the rod below the hand (B), or two 50-g masses affixed to the ends of the rod above and below the hand $(C)$.

partial length, the magnitude of the rod above the hand is identical in the no-added-mass and the one-added-mass conditions. Thus the perceived partial lengths should be similar in these two conditions. In contrast, the perceived partial lengths for the two-added-mass condition should be greater than either the no- or one-added-mass condition, because of the increase in the magnitude of that portion of the object (see Solomon \& Turvey, 1988; Solomon et al., 1989b). Thus perceived partial length as a function of the added-mass condition should appear similar to the function depicted in Figure 3B. In short, the expectation that the perceptions of partial length and hand position depend differently on $I_{i j}$ is expressed most simply as an expectation of an added mass $\times$ task interaction of the type depicted in Figure 3.

\section{Method}

Observers. Ten undergraduate students ( 6 men and 4 women) at the University of Connecticut participated in Experiment 1 in partial fulfillment of course requirements. All 10 observers were right handed.

Materials and Apparatus. The wielded objects were three cylindrical wooden rods, $60 \mathrm{~cm}$ in length and $1.2 \mathrm{~cm}$ in diameter, weighing $42 \mathrm{~g}$. A mass was added to one end of one of the rods, a mass was added to each end of another rod, and no mass was added to the remaining rod. The added masses consisted of a 50-g metal disk (outside radius $=1.6 \mathrm{~cm}$; inside radius $=.63 \mathrm{~cm}$; height $=.9 \mathrm{~cm}$ ). Table 2 presents the $I_{i j}$ for the objects. The experimental arrangement is depicted in Figure 4, and was similar to that used by Pagano et al. (1994). The observer sat with his or her right forearm resting on a horizontal surface attached to a seat, with the wrist extending approximately $2 \mathrm{~cm}$ from the edge of the surface. A black curtain occluded the observer's view of his or her right hand. A visible 60-cm report rod was mounted vertically in front of the observer on a low table. The height of the table was such that the full length of the report rod could be reached easily by all of the observers.

Procedure. In each trial, one of the three $60-\mathrm{cm}$ rods was placed in the observer's right hand at one of three positions, with 15,30 , or $45 \mathrm{~cm}$ of the total rod length extending above the hand (with measurements taken from the center of the hand). Observers wielded the objects using movements about the wrist only, keeping the forearm on the horizontal surface and grasping the rod firmly within the closed fist so that it did not move within the hand. Thus, although motions were restricted to those about the wrist, observers were allowed, and appeared to employ, the full range of three-space motions about that point. In half of the trials, the observer was asked to position his or her left hand along the visible report rod so that the length of the report rod above the left hand corresponded to the felt length of the part of the wielded rod above the right hand (hereafter referred to as "length" trials). In the remaining trials, the observer was asked to locate his or her left hand along the visible report rod so that it corresponded to the felt position of the right hand on the wielded object, where "position" was defined as the proportional position along the rod (hereafter referred to as "position" trials). Each observer was allowed to wield the rod and to adjust his or her left hand on the 60 -cm report rod for as long as desired until satisfied with the judgment.

No practice or feedback was given, and observers were not told the number or lengths of rods used or that the rods might be weighted. The combination of 3 mass conditions, 3 hand positions, and 2 wielding intentions resulted in 18 different wielding conditions. Each condition was presented three times for a total of 54 trials per observer. The length and position trials were each split into two blocks for a total of four blocks. Half of the observers wielded to perceive length in the first and third blocks and wielded to perceive position in the second and fourth blocks; the other half wielded to perceive position in the first and third blocks and to perceive length in the second and fourth blocks. Within each block, the grip position $\times$ mass conditions were presented to the observer in random order, with all conditions being run once before being repeated.

\section{Results and Discussion}

The results are summarized in Table 2 . Figure 5 provides a compact overview of the experimental data in respect to the manipulations of added mass and task. So that the results from the two tasks can be compared directly, both perceptual measures are presented in Figure 5 in terms of the distance from the hand on the report rod to the report rod's uppermost end. The expectation that the perceptions of partial length and hand position
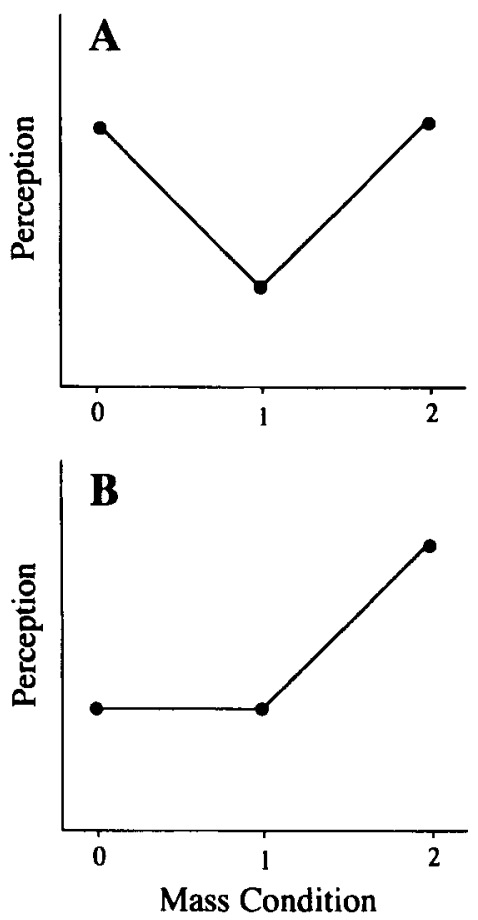

Figure 3. Expected perceived hand position (A) and expected mean perceived partial rod length $(B)$ as functions of the added mass. Both perceptual measures are the distance from the hand on the report rod to the rod's uppermost end. 
Table 2

Actual Partial Rod Lengths, Actual Grip Positions, $\boldsymbol{I}_{i j}$, Perceived Partial Length, and Perceived Grip Position as a Function of the Added-Mass Conditions Used in Experiment 1

\begin{tabular}{|c|c|c|c|c|c|c|c|}
\hline \multirow{2}{*}{$\begin{array}{c}\text { Actual } \\
\text { Partial } \\
\text { Length }(\mathrm{cm})^{*}\end{array}$} & \multirow{2}{*}{$\begin{array}{c}\text { Actual } \\
\text { Grip } \\
\text { Position } \dagger\end{array}$} & \multicolumn{4}{|c|}{$I_{i j}\left(\mathrm{~g} \cdot \mathrm{cm}^{2 / 1}, 000\right)$} & \multirow{2}{*}{$\begin{array}{c}\text { Perceived } \\
\text { Partial } \\
\text { Length }(\mathrm{cm})^{*}\end{array}$} & \multirow{2}{*}{$\begin{array}{l}\text { Perceived } \\
\text { Grip } \\
\text { Position } \dagger\end{array}$} \\
\hline & & $I_{x x}$ & $I_{y y}$ & $I_{z z}$ & $I_{y z}$ & & \\
\hline \multicolumn{8}{|c|}{ No Added Mass } \\
\hline 15.00 & .25 & 26.09 & 1.68 & 24.42 & 4.18 & 16.0 & .32 \\
\hline 30.00 & .50 & 15.63 & 1.68 & 13.95 & 0 & 21.7 & .50 \\
\hline 45.00 & .75 & 26.09 & 1.68 & 24.42 & -4.18 & 34.3 & .67 \\
\hline \multicolumn{8}{|c|}{ One Added Mass } \\
\hline 15.00 & .25 & 122.90 & 3.34 & 119.64 & 17.73 & 17.8 & .24 \\
\hline 30.00 & .50 & 58.71 & 3.34 & 55.45 & 8.76 & 15.9 & .31 \\
\hline 45.00 & .75 & 35.29 & 3.34 & 32.03 & .61 & 27.7 & .50 \\
\hline \multicolumn{8}{|c|}{ Two Added Masses } \\
\hline 15.00 & .25 & 136.51 & 5.19 & 131.47 & 12.59 & 21.4 & .30 \\
\hline 30.00 & .50 & 105.03 & 5.19 & 99.99 & 0 & 31.7 & .51 \\
\hline 45.00 & .75 & 136.51 & 5.19 & 131.47 & -12.59 & 43.8 & .75 \\
\hline
\end{tabular}

Note $-I_{x y}=I_{x z}=0 . \quad$ *Length of the rod above the hand $(\mathrm{cm})$. †Proportion of rod length above the hand: partial rod length $(\mathrm{cm}) /$ whole rod length $(\mathrm{cm})$.

depend differently on $I_{i j}$ is expressed most simply as the expectation of an added mass $\times$ task interaction. Inspection of Table 2 reveals that mean $I_{y z}$ is identical $\left(0 \mathrm{~g} \mathrm{~cm}^{2} \times 10^{-3}\right)$ in both the zero- and two-added-mass conditions. Consequently, the expectation is that perceived hand position should be identical for the zero- and two-added-mass conditions. Further inspection of Table 2 reveals that all three moments of inertia increase with the addition of a single mass, and then increase again with the addition of a second added mass. This increase in moments of inertia leads to the expectation that the perceived partial lengths for the three conditions should be different. An interaction of the expected kind (Figure 3 ) between added mass and perceptual task is suggested in Figure 5 and was confirmed by an analysis of variance (ANOVA) $[F(2,18)=7.85, p<.01]$.

Perceived grip position. Overall, the hand was perceived to be positioned on the $60-\mathrm{cm}$ rod with $49.4 \%$,

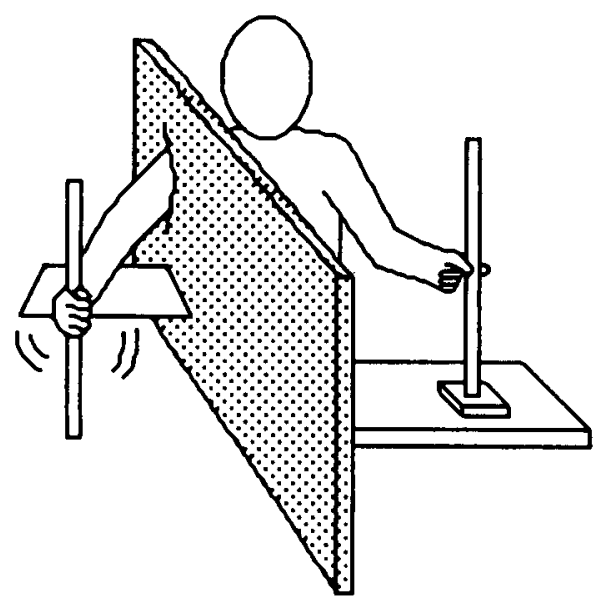

Figure 4. The apparatus used in the experiments.
$37.1 \%$, and $49.5 \%$ of the total rod length (i.e., $29.4,22.3$, and $29.7 \mathrm{~cm}$ ) extending above the hand in the no-addedmass, one-added-mass, and two-added-mass conditions, respectively. The hand was perceived to be positioned with $28.7 \%, 43.6 \%$, and $63.7 \%$ of the total rod length (i.e., 17.2, 26.2, and $38.2 \mathrm{~cm}$ ) extending above the hand in the $25 \%, 50 \%$, and $75 \%$ (i.e., $15-, 30-$, and $45-\mathrm{cm}$ ) grip position conditions, respectively. A $3 \times 3$ ANOVA with within-subject factors of added-mass condition and grip position confirmed a significant main effect for added mass $[F(2,18)=9.3, p<.005]$ and grip position $[F(2,18)=$ $104.3, p<.0001]$, as well as a significant mass $\times$ grip position interaction $[F(4,36)=4.8, p<.005]$. As predicted, the interaction was due to the perceived grip position values more closely matching the actual grip positions in the no- and two-added-mass conditions as compared with the one-added-mass condition. The perceived grip position values in the one-added-mass condition were closest to the values in the other conditions when the hand was nearer to the top of the rod (Table 2). A Tukey honestly significant difference (HSD) test confirmed that the mean perceived grip position was higher in the condition with one added mass compared to the conditions with either two or no added mass (both $p s<.01$ ). Perceived grip positions were similar in the conditions with two or no added mass $(p>.05)$. This outcome indicates that perceived position of grasp varied as a function of the way the mass of the entire rod is distributed relative to the hand. Multiple regression predicting mean perceived grip position from $I_{x x}, I_{y y}$, and $I_{y z}\left(I_{z z} \approx I_{x x}, I_{x y}=I_{x z}=\right.$ 0 ) resulted in an $r^{2}=.90$, with only $I_{y z}$ significant after backwards elimination. ${ }^{5}$ For the 10 individual subjects, this multiple regression resulted in $r^{2} \mathrm{~s}$ of $.94, .89, .90$, $.93, .77, .91, .89, .89, .84$, and .88 .

Perceived partial length. Overall, the length of the portion of the rod extending above the hand was perceived to be $24.0,21.9$, and $31.0 \mathrm{~cm}$ for the no-added- 


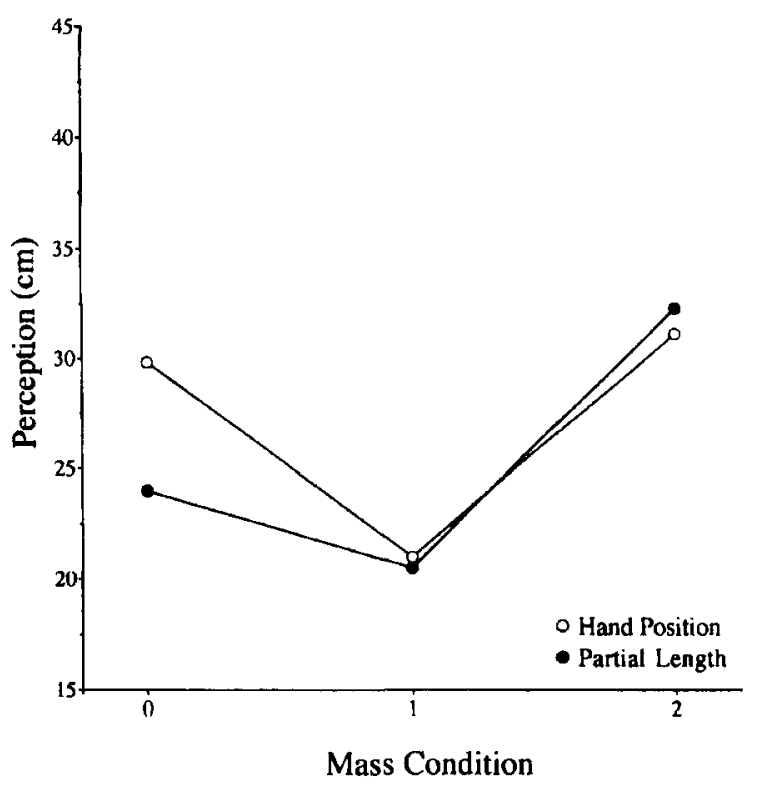

Figure 5. Mean perceived hand position and mean perceived partial rod length as functions of the added mass variable in Experiment 1 . Both perceptual measures are the distance from the hand on the report rod to the rod's uppermost end.

mass, one-added-mass, and two-added-mass conditions, respectively, and $18.7,23.0$, and $35.2 \mathrm{~cm}$ for the hand positioned with 15,30 , and $45 \mathrm{~cm}$ extending above the hand, respectively. A $3 \times 3$ ANOVA with within-subject factors of mass condition and hand position confirmed a significant main effect for mass $[F(2,18)=6.2, p<.01]$ and hand position $[F(2,18)=49.3, p<.0001]$, as well as a significant mass $\times$ hand position interaction $[F(4,36)=$ $7.0, p<.001]$. The interaction was due to the perceived partial length values in the one-added-mass condition remaining constant between the .25 and .50 grip positions (Table 2). A Tukey HSD test confirmed that mean perceived partial length was greater in the condition with two added masses compared to the conditions with either one or zero added mass (both $p<.01$ ), and were similar in the one- and zero-added-mass conditions $(p>.05)$. This outcome indicates that perceived partial length was more affected by the magnitude of the object's mass located above the hand than by the magnitude of the object's mass located below the hand. Multiple regression predicting mean perceived partial length from $I_{x x}, I_{y y}$, and $I_{y z}\left(I_{z z} \approx I_{x x}, I_{x y}=I_{x z}=0\right)$ resulted in $r^{2}=.89$, with $I_{x x}$ and $I_{y z}$ significant after backwards elimination [partial $F_{\mathrm{S}}=9.83(p<.02)$ and $44.67(p<.001)$ for $I_{x x}$ and $I_{y z}$, respectively]. For the 10 individual subjects, this multiple regression resulted in $r^{2} \mathrm{~s}$ of $.93, .81, .78, .73, .73$, $.97, .92, .92, .84$, and .81 .

In sum, the results of Experiment 1 are consonant with the hypothesis that the components of $I_{i j}$ for an entire hand-held object can support both the perception of grip position and the perception of the magnitude of the part of the object that lies in a particular direction (forward or aft) relative to the hand. The results further indicate that the way in which $I_{i j}$ supports perception is different for these two properties. Perceived grip position is a function of an off-diagonal component, a product of $I_{i j}$, whereas perceived partial length is a function of diagonal and offdiagonal components, moments and products of $I_{i j}$, respectively.

\section{EXPERIMENT 2}

A second experiment was conducted replicating $\mathrm{Ex}$ periment 1 in all respects except for the length of the report rod. The report rod in Experiment 1 was equal in length to the wielded rod. If observers perceived the partial length above the hand to be in excess of that permitted by the report rod, they would have been forced to give a response that underestimated their perception. To accommodate this possibility, Experiment 1 was repeated using a report rod of $100 \mathrm{~cm}$.

\section{Method}

Observers. Ten graduate students ( 5 men and 5 women) at the University of Connecticut participated in Experiment 2 on a volunteer basis. One observer misunderstood the directions to the experiment, and was replaced.

Materials and Apparatus. The apparatus used in Experiment 2 was identical to that used in Experiment 1, with the exception that the report rod was $100 \mathrm{~cm}$ long instead of $60 \mathrm{~cm}$. Objects of the same dimensions as those used in Experiment 1 were constructed. Table 3 presents the $I_{i j}$ for the objects used in Experiment 2; because of small variations in wood density, these differ slightly from those used in Experiment 2.6

Procedure. The procedure used was identical to that of Experiment 1 , with the exception that all observers wielded to perceive length in the first and third blocks and wielded to perceive position in the second and fourth blocks.

\section{Results and Discussion}

The results are presented in Table 3. Figure 6 summarizes the data in respect to the manipulations of added mass and task. Again, the expectation that the perceptions of partial length and hand position depend differently on $I_{i j}$ is expressed most simply as the expectation of an added mass $\times$ task interaction. This interaction can be seen in Figure 6 and was confirmed by an ANOVA $[F(2,18)=43.40, p<.0001]$.

Perceived grip position. Overall, the hand was perceived to be positioned with $52.0 \%, 38.3 \%$, and $52.9 \%$ of the total rod length (i.e., $31.2,23.0$, and $31.7 \mathrm{~cm}$ ) extending above the hand in the no-added-mass, oneadded-mass, and two-added-mass conditions, respectively. The hand was perceived to be positioned with $32.2 \%$, $48.5 \%$, and $62.5 \%$ of the total rod length (i.e., 19.3, 29.1, and $37.5 \mathrm{~cm}$ ) extending above the hand in the $25 \%, 50 \%$, and $75 \%$ grip position conditions, respectively. A $3 \times 3$ ANOVA with within-subject factors of mass condition and grip position confirmed a significant main effect for mass $[F(2,18)=43.6, p<.0001]$ and grip position $[F(2,18)=83.0, p<.0001]$, as well as a significant mass $\times$ grip position interaction $[F(4,36)=9.5, p<.0001]$. 
Table 3

Actual Partial Rod Lengths, Actual Grip Positions, $\boldsymbol{I}_{\boldsymbol{i}}$,

Perceived Partial Length, and Perceived Grip Position as a

Function of the Added-Mass Conditions Used in Experiment 2

\begin{tabular}{|c|c|c|c|c|c|c|c|}
\hline \multirow{2}{*}{$\begin{array}{c}\text { Actual } \\
\text { Partial } \\
\text { Length }(\mathrm{cm})^{*}\end{array}$} & \multirow{2}{*}{$\begin{array}{c}\text { Actual } \\
\text { Grip } \\
\text { Position } \dagger\end{array}$} & \multicolumn{4}{|c|}{$I_{i j}\left(\mathrm{~g} \cdot \mathrm{cm}^{2} / 1,000\right)$} & \multirow{2}{*}{$\begin{array}{c}\text { Perceived } \\
\text { Partial } \\
\text { Length }(\mathrm{cm})^{*}\end{array}$} & \multirow{2}{*}{$\begin{array}{c}\text { Perceived } \\
\text { Grip } \\
\text { Position } \dagger\end{array}$} \\
\hline & & $I_{x x}$ & $I_{y y}$ & $I_{z z}$ & $I_{y z}$ & & \\
\hline \multicolumn{8}{|c|}{ No Added Mass } \\
\hline 15.00 & .25 & 25.08 & 1.62 & 23.47 & 4.02 & 17.7 & .38 \\
\hline 30.00 & .50 & 15.63 & 1.62 & 13.41 & 0 & 21.1 & .54 \\
\hline 45.00 & .75 & 25.08 & 1.62 & 23.47 & -4.02 & 26.8 & .64 \\
\hline \multicolumn{8}{|c|}{ One Added Mass } \\
\hline 15.00 & .25 & 129.84 & 3.50 & 126.42 & 17.73 & 23.0 & .26 \\
\hline 30.00 & .50 & 62.57 & 3.50 & 59.15 & 9.18 & 24.2 & .34 \\
\hline 45.00 & .75 & 38.04 & 3.50 & 34.62 & .63 & 30.4 & .55 \\
\hline \multicolumn{8}{|c|}{ Two Added Masses } \\
\hline 15.00 & .25 & 139.78 & 5.28 & 134.65 & 12.94 & 31.0 & .32 \\
\hline 30.00 & .50 & 107.16 & 5.28 & 102.02 & 0 & 42.0 & .58 \\
\hline 45.00 & .75 & 138.70 & 5.28 & 133.57 & -12.73 & 52.3 & .69 \\
\hline
\end{tabular}

Note $-I_{x y}=I_{x z}=0 . \quad$ *Length of the rod above the hand $(\mathrm{cm}) . \quad$ 'Proportion of rod length above the hand: partial rod length $(\mathrm{cm}) /$ whole rod length $(\mathrm{cm})$.

As predicted, the interaction was due to the perceived grip position values more closely matching the actual grip positions in the no- and two-added-mass conditions, as compared with the one-added-mass condition (Table 3 ). A Tukey HSD test confirmed that the mean perceived grip position was higher in the condition with one added mass compared with the conditions with either two or no added mass (both $p s<.01$ ). Perceived grip positions were similar in the conditions with two or no added mass $(p>.05)$. This outcome indicates that perceived posi-

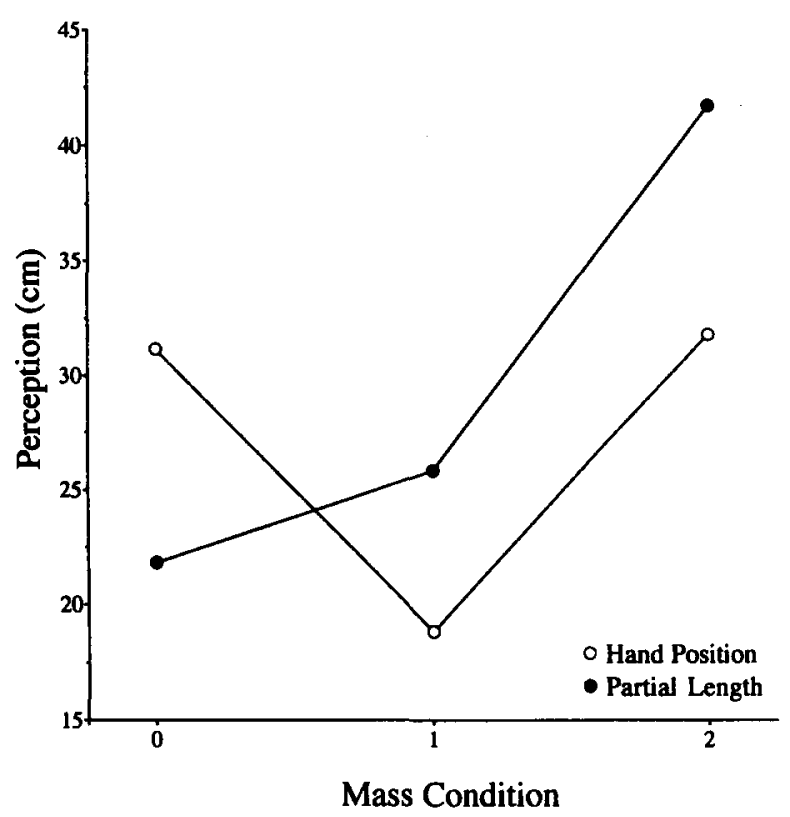

Figure 6. Mean perceived hand position and mean perceived partial rod length as functions of the added mass variable in $\mathbf{E x}-$ periment 2. Both perceptual measures are the distance from the hand on the report rod to the rod's uppermost end. tion of grasp varied as a function of the way the mass of the entire rod was distributed relative to the hand. Multiple regression predicting mean perceived grip position from $I_{x x}, I_{y y}$, and $I_{y z}\left(I_{z z} \approx I_{x x}, I_{x y}=I_{x z}=0\right)$ resulted in $r^{2}=.92$ with only $I_{y z}$ significant after backwards elimination. For the 10 individual subjects, this multiple regression resulted in $r^{2} \mathrm{~s}$ of $.90, .84, .91, .90, .95, .92, .84$, $.88, .79$, and .87 . Figure 7 shows the close similarity between the results of Experiments 1 and 2. It also makes clear that (1) the perception of hand position was minimally affected by $I_{x x}$ in the two experiments and (2) the dependency on $I_{y z}$ was nonlinear, meaning that the linear regression analyses of the two experiments underestimated the degree of dependence.

Perceived partial length. Overall, the length of the portion of the rod extending above the hand was perceived to be $21.9,25.9$, and $41.7 \mathrm{~cm}$ for the no-addedmass, one-added-mass, and two-added-mass conditions, respectively, and $23.9,29.1$, and $36.5 \mathrm{~cm}$ for the hand in the $25 \%, 50 \%$, and $75 \%$ hand position conditions, respectively. A $3 \times 3$ ANOVA with within-subject factors of mass condition and hand position confirmed a significant main effect for mass $[F(2,18)=95.7, p<.0001]$ and hand position $[F(2,18)=18.4, p<.0001]$, as well as a significant mass $\times$ hand position interaction $[F(4,36)=$ $11.5, p<.0001]$. The interaction was due to the perceived partial length values in the two-added-mass condition increasing with actual partial length at a higher rate than in either of the other mass conditions (Table 3 ). A Tukey HSD test confirmed that mean perceived partial length was greater in the condition with two added masses compared with the conditions with either one or no added mass (both $p s<.01$ ), whereas perceived partial lengths were greater in the one-added-mass condition compared with the no-added-mass condition $(p<.05)$. This outcome indicates that perceived partial length corresponded to the magnitude of the object's mass located above the hand 

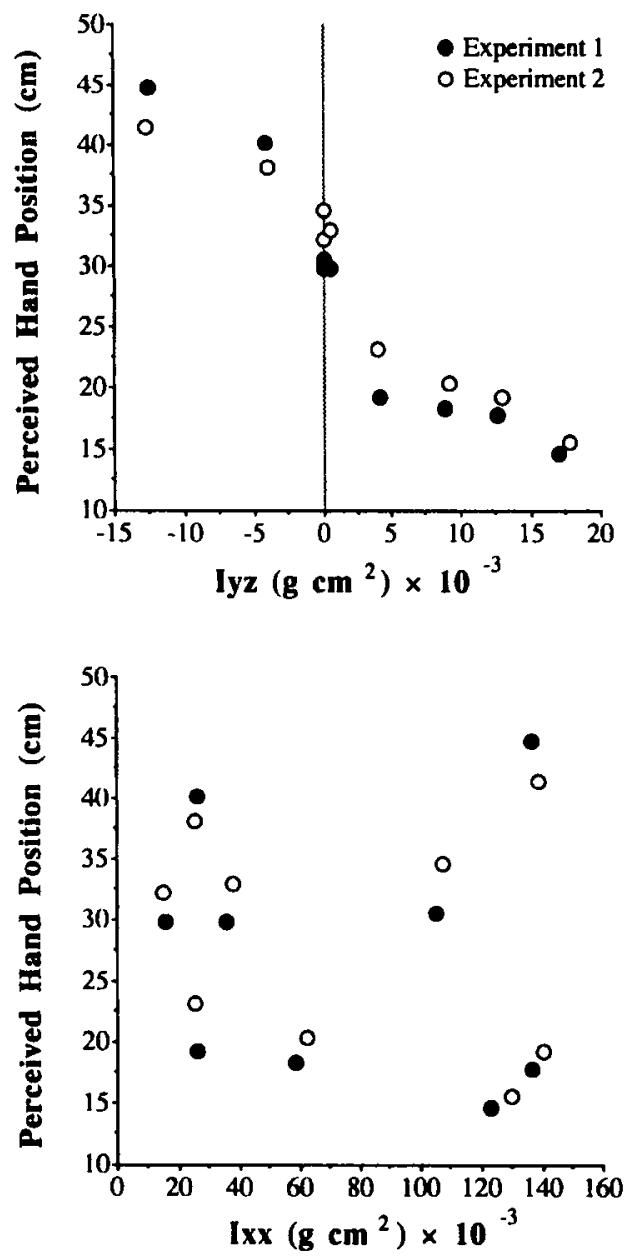

Figure 7. Perceived hand position as a function of $I_{y z}$ (upper) and $I_{x x}$ (lower) in Experiments 1 and 2.

to a greater extent than the magnitude of the object's mass located below the hand. Multiple regression predicting mean perceived partial length from $I_{x x}, I_{y y}$, and $I_{y z}\left(I_{z z} \approx\right.$ $I_{x x}, I_{x y}=I_{x z}=0$ ) resulted in an $r^{2}=.99$, with $I_{x x}, I_{y y}$, and $I_{y z}$ significant [partial $F \mathrm{~s}=7.3(p<.05), 24.8(p<$ $.005)$, and $157.4(p<.0001)$ for $I_{x x}, I_{y y}$, and $I_{y z}$, respectively]. For the 10 individual subjects, this multiple regression resulted in $r^{2} \mathrm{~s}$ of $.95, .95, .98, .99, .93, .95, .92$, $.97, .84$, and .91 .

Comparison of Figures 5 and 6 suggests that the increase in the length of the report rod from Experiment 1 to Experiment 2 had an effect. The mean perceived partial length in Experiment 2 was $29.8 \mathrm{~cm}$ (for an actual mean value of $30 \mathrm{~cm}$ ) compared with $25.6 \mathrm{~cm}$ in Experiment 1 , a difference that was almost significant $[F(1,18)=3.7$, $p=.07]$. A clearer effect is seen in the important interaction between the added mass manipulation and experiment $[F(2,36)=8.7, p<.001]$. Considering the $r^{2}$ values on the multiple regressions (.99 in Experiment 2 vs. .89 in Experiment 1), it seems that the longer report rod allowed the observers in Experiment 2 to provide more accurate reports of their impressions of partial length, in the sense that a greater proportion of the variance could be accounted for by $I_{i j}$.

In sum, the results of Experiment 2 replicated the main findings of Experiment 1; the components of $I_{i j}$ for an entire hand-held object were found to support the perception of grip position, as well as perception of the magnitude of the part of the object that lies in a particular direction relative to the hand. Similarly, the results indicate that the way in which $I_{i j}$ supports perception is different for these two properties: Perceived grip position is specific to only the products of inertia, whereas perceived partial length is specific to both the moments and the products.

\section{GENERAL DISCUSSION}

When an object is grasped firmly and wielded (i.e., hefted, swung, carried, or otherwise manipulated), the hand movements together with the physical properties of the object produce an array of torques affecting the tensile states of muscles and tendons in the hand and arm. Muscle spindles and Golgi tendon organs constitute the receptor foundation for the haptic sensitivity to such torques, and are collectively referred to as the "muscle sense" (see, e.g., Bell, 1826; Clark \& Horch, 1986; Shepherd, 1988). The haptic subsystem of dynamic touch is characterized primarily by this muscular sense - more so than by patterns of neural activity arising from skin deformations (cutaneous touch) or changes in joint angle. Although research involving the muscle sense has traditionally focused on its proprioceptive role, the present investigation is part of a recent body of work directed at its abilities to register object properties and the orientation of the grasping hand to an object's mass distribution (for a review, see Turvey \& Carello, 1995; see also Fitzpatrick et al., 1994; Pagano \& Turvey, 1995; Turvey, 1994, for discussions of the muscle sense and its role in dynamic touch). A major concern of this work has been to answer the question: What properties of a visually occluded object and of the hand's relation to that object can an observer apprehend through dynamic touch? The physics involved in producing the requisite torques demand that such properties be tied to mechanical variables - which are based on the dimension mass (e.g., force, inertia, kinetic energy; see Solomon, 1988; Solomon \& Turvey, 1988). That is, unless an object property is associated with one or more mechanical variables, there is no mechanism by which that property may systematically affect the array of torques upon which perception is based. Thus color is an obvious example of an object property not perceptible by dynamic touch. Less obvious, however, is the status of geometric properties such as object length or orientation in space. The present work, along with the accumulated evidence of past research, indicates that aspects of an object's overall mass distribution can be detected. Additionally, this work has identified $I_{i j}$, an invariant rendering of the persistent mass distribution of the entire hand-held object, as the relevant independent variable for object properties and hand-object relations perceived by dynamic touch. 
As outlined in the introduction, $I_{i j}$ quantifies the object's resistance to rotational acceleration that occurs in different directions (tangential and normal to the angular rotations about each of the three spatial axes) with different magnitudes in these different directions, and is specific to the manner in which the object's mass is distributed about the axes. $I_{i j}$ is a quantity with the dimensions of mass $\times$ length $^{2}$, therefore, properties such as length and weight can affect the muscles and tendons only by virtue of their contribution to $I_{i j}$ (see, e.g., Amazeen \& Turvey, 1996; Solomon \& Turvey, 1988). Likewise, perceived object "shape" matches the components of $I_{i j}$ measured in different directions about the point of rotation, and does not match all properties of object shape that are salient visually (Burton et al., 1990). Similarly, observers asked to wield an L-shaped rod consisting of a branch attached perpendicularly at the distal end of a hand-held stem are able to report the orientation of the branch in space (Turvey et al., 1992). Perception of object orientation is possible because of the object's mass distribution, as quantified by $I_{i j}$, varying systematically with changes in branch direction. However, with two branches forming a $V$ attached perpendicularly to the distal end of the stem, observers are unable to report the geometric orientation of one branch taken individually, but rather, are sensitive to the orientation of $I_{i j}$ for the entire object (Pagano \& Turvey, 1992). In the present set of experiments, the properties of grip position and partial rod length were found to be perceptible, with these separate impressions being based on separate aspects of $I_{i j}$. Specifically, perceived grip position was found to be a function of an off-diagonal term, a product of $I_{i j}$, whereas perceived partial length was a function of both a diagonal and an off-diagonal term. In sum, perception of the properties in question is possible because of the manner in which $I_{i j}$ reflects the mass distribution of the whole object relative to the hand, as well as the proportional distribution of this mass that is located to one side of the hand.

Patently, perceived grip position does not necessarily go as actual grip position; likewise, perceived partial length does not go as actual partial length (when actual position and length are measured in purely geometric terms). In the handling of objects, exact perception of grasp relative to the linear dimensions of the object (e.g., its lengths) is of less importance than exact perception of grasp relative to the mass distribution of the object. The torques provided through muscular forces acting on linked segments to guide and steer a hand-held object must be scaled to how the object's mass lies in reference to the momentary fixed point of the object's motions. What the products of $I_{i j}$ specify is hand position relative to CM. What the moments and products of inertia specify is the magnitudes of the object's mass distribution about any given axes of rotation, with these magnitudes being of the dimensions mass $\times$ length ${ }^{2}$ - the object dimensions of primary relevance in the dynamics of rotation.

As noted in the introduction, when one grasps and wields an occluded object, there is conjoint perception of aspects of the object's magnitudes, perception of how the body segments are oriented relative to each other and to the body, and perception of how the object is oriented relative to the body segments, and vice versa. Following Lee (1978; Lee \& Lishman, 1977), the three kinds of perceiving correspond to three kinds of information; specifically, exterospecific information (about environmental surfaces, objects, and events), propriospecific information (about the positions and movements of the limbs relative to each other and to the body), and expropriospecific information (about the position, orientation, or movement of the body as a whole, or a part of the body, relative to the environment). In the case of visual perception and the control of locomotion, Gibson (1979) and Lee (1978) have laid the groundwork for the argument that the three information kinds may be identified with mathematically distinct aspects of the flow field visually available to the moving observer. Gibson and Lee described the flow field in optical terms, as a transforming structured array of different light intensities in different directions. In the present research, we investigated the possibility that exteroception and exproprioception are tied to mathematically distinct aspects of the structured array of resistances to rotational acceleration present in the use of hand-held implements. As noted, the results indicate that perceived partial rod length and perceived grip position depend in different ways on $I_{i j}$.

Like the optic array, $I_{i j}$ is a structured array of intensities; it is composed of different intensities in different directions. Importantly, $I_{i j}$ is sufficiently structured to be simultaneously informative about distinct object properties, such as an object's overall magnitude (see, e.g., Solomon \& Turvey, 1988), its orientation (see, e.g., Pagano \& Turvey, 1992), and its position relative to the hand (Pagano et al., 1994). The present results indicate the capability of selective sensitivity to different aspects of $I_{i j}$-those specific to partial rod magnitude and grip position (see also Burton \& Turvey, 1991).

In closing, it should be noted that $I_{i j}$ is one of many movement constraints of relevance to dynamic touch. In situations where the relevant dynamics are characterized by the wielding of a hand-held object (Solomon \& Turvey, 1988; the present work) or limb (Pagano \& Turvey, 1995) in space, the inertia tensor will suffice. Other movementproduced invariants come into play, however, when they are required to more fully characterize the dynamicssuch as the center of percussion of a hand-held object struck against an environmental surface (Carello, Fitzpatrick, \& Turvey, 1992; Chan \& Turvey, 1991), or the collective parameter Lambda for a limb + object configuration used to probe a gap (Barac-Cikoja \& Turvey, 1991, 1993, 1995). Observers may capitalize on these additional parameters to make salient object properties not revealed by wielding alone. Additionally, a general explanation of dynamic touch's ability to selectively perceive one of two things in the hand may involve attitude spinors-mathematical objects (rotational operators) that can act in conjunction with $I_{i j}$ when the observer selectively attends to one of two object components (Turvey et al., 
1996). The relation between such invariants and $I_{i j}$ in exteroceptive, proprioceptive, and exproprioceptive tasks will be an important topic for continuing study.

\section{REFERENCES}

Amazeen, E. L., \& Turvey, M. T. (1996). Weight perception and the haptic "size-weight illusion" are functions of the inertia tensor. Journal of Experimental Psychology: Human Perception \& Performance, 22, 213-232.

Barac-Cikoja, D., \& Turvey, M. T. (1991). Perceiving aperture size by striking. Journal of Experimental Psychology: Human Perception \& Performance, 17, 330-346.

BaraC-Cikoja, D., \& Turvey, M. T. (1993). Haptically perceiving size at a distance. Journal of Experimental Psychology: General, 122, $347-370$.

Barac-Cikoja, D., \& Turvey, M. T. (1995). Does perceived size depend on perceived distance? An argument from extended haptic perception. Perception \& Psychophysics, 57, 216-224.

BELL, C. (1826). On the nervous circle which connects the voluntary muscles with the brain. Philosophical Transactions of the Royal Society, 116, 163-173

Burgess, P. R., WEI, J. Y., Clark, F. J., \& Simon, J. (1982). Signaling of kinesthetic information by peripheral sensory receptors. Annual Review of Neuroscience, 5, 171-187.

Burton, G., \& Turvey, M. T. (1990). Perceiving the lengths of rods that are held but not wielded. Ecological Psychology, 2, 295-324.

Burton, G., \& TURvey, M. T. (1991). Attentionally splitting the mass distribution of hand-held rods. Perception \& Psychophysics, 50, 129140.

Burton, G., Turvey, M. T., \& Solomon, H. Y. (1990). Can shape be perceived by dynamic touch? Perception \& Psychophysics, 48, 477-487.

Carello, C., Fitzpatrick, P., Domaniewicz, I., Chan, T.-C., \& TurVEY, M. T. ( 1992). Effortful touch with minimal movement. Journal of Experimental Psychology: Human Perception \& Performance, 18. 290-302.

Carello, C., Fitzpatrick, P., \& Turvey, M. T. (1992). Haptic probing: Perceiving the length of a probe and the distance of a surface probed. Perception \& Psychophysics, 51, 580-598

Carello, C., Santana, M.-V., \& Burton, G. (1996). Selective perception by dynamic touch. Perception \& Psychophysics, 58, 1177-1190.

CHAN, T.-C. (1994). Haptic perception of partial-rod lengths with the rod held stationary or wielded. Perception \& Psychophysics, 55, 551561.

Chan, T.-C., \& Turvey, M. T. (1991). Perceiving the vertical distances of surfaces by means of a hand-held probe. Journal of Experimental Psychology: Human Perception \& Performance, 17, 347-358.

Clark, F. J., \& Horch, K. W. (1986). Kinesthesia. In K. R. Boff, L. Kaufman, \& J. P. Thomas (Eds.), Handbook of perception and human performance (Vol. 1, pp. 13-1 to 13-62). New York: Wiley.

DEN HaRToG, J. P. (1961). Mechanics. New York: Dover. (Original work published 1948)

Fitzpatrick, P., Carello, C., \& Turvey, M. T. (1994). Eigenvalues of the inertia tensor and exteroception by the "muscular sense." Neuroscience, 60, 551-568.

GiBsON, J. J. (1966). The senses considered as perceptual systems. Boston: Houghton Mifflin.

GiBSon, J. J. (1979). The ecological approach to visual perception. Boston: Houghton Mifflin.

Goldstein, H. (1980). Classical mechanics. Reading, MA: Addison Wesley.

KELSO, J. A. S. (1978). Joint receptors do not provide a satisfactory basis for motor timing and positioning. Psychological Review, 85, 474-481.

Kibble, T. W. B. (1985). Classical mechanics. London: Longman.

Lederman, S. J., Ganeshan, S. R., \& Ellis, R. E. (1996). "Effortful touch with minimum movement" revisited. Journal of Experimental Psychology: Human Perception \& Performance, 22, 851-868.

LEE, D. N. (1978). The functions of vision. In H. L. Pick, Jr., \& E. Saltzman (Eds.), Modes of perceiving and processing information (pp. 159-170). Hillsdale, NJ: Erlbaum.
LeE, D. N., \& Lishman, R. (1977). Visual control of locomotion. Scandinavian Journal of Psychology, 18, 224-230.

LoOMis, J. M., \& Lederman, S. J. (1986). Tactual perception. In K. R. Boff, L. Kaufman, \& J. P. Thomas (Eds.), Handbook of perception and human performance (Vol. 1, pp. 31-1 to 31-41). New York: Wiley.

LovetT, D. R. (1989). Tensor properties of crystals. Philadelphia: Adam Hilger.

MatT HEWs, P. B. C. (1982). Where does Sherrington's "muscular sense" originate? Muscles, joints, corollary discharges? Annual Review of Neuroscience, 5, 189-218.

McCloskey, D. I. (1978). Kinesthetic sensibility. Psychological Reviews, 58, 763-820.

MOON, P., \& SPENCER, D. E. (1986). Theory of holors. Cambridge: Cambridge University Press.

Pagano, C. C., Fitzpatrick, P., \& Turvey, M. T. (1993). Tensorial basis to the constancy of perceived object extent over variations of dynamic touch. Perception \& Psychophysics, 54, 43-54.

Pagano, C. C., Garrett, S. R., \& Turvey, M. T. (1996). Is limb proprioception a function of the limbs' inertial eigenvectors? Ecological Psychology, 8, 43-69.

Pagano, C. C., Kinsella-Shaw, J. M., Cassidy, P. E., \& Turvey, M. T. (1994). Role of the inertia tensor in haptically perceiving where an object is grasped. Journal of Experimental Psychology: Human Perception \& Performance, 20, 276-285.

Pagano, C. C., \& Turvey, M. T. (1992). Eigenvectors of the inertia tensor and perceiving the orientation of a hand-held object by dynamic touch. Perception \& Psychophysics, 52, 617-624.

Pagano, C. C., \& Turvey, M. T. (1993). Perceiving by dynamic touch the distances reachable with irregular objects. Ecological Psychology, 5, 125-151.

Pagano, C. C., \& Turvey, M. T. (1995). The inertia tensor as a basis for the perception of limb orientation. Journal of Experimental Psychology: Human Perception \& Performance, 21, 1070-1087.

Shepherd, G. M. (1988). Neurobiology. New York: Oxford University Press.

ShERrington, C. (1906). The integrative action of the nervous system. New Haven, CT: Yale University Press.

Solomon, H. Y. (1988). Movement-produced invariants in haptic explorations: An example of a self-organizing, information-driven, intentional system. Human Movement Science, 7, 201-224.

Solomon, H. Y., \& Turvey, M. T. (1988). Haptically perceiving the distances reachable with hand-held objects. Journal of Experimental Psychology: Human Perception \& Performance, 14, 404-427.

Solomon, H. Y., Turvey, M. T., \& BurTon, G. (1989a). Gravitational and muscular variables in perceiving rod extent by wielding. Ecological Psychology, 1, 265-300.

Solomon, H. Y., Turvey, M. T., \& Burton, G. (1989b). Perceiving extents of rods by wielding: Haptic diagonalization and decomposition of the inertia tensor. Journal of Experimental Psychology: Human Perception \& Performance, 15, 58-68.

Turvey, M. T. (1994), From Borelli (1680) and Bell (1826) to the dynamics of action and perception. Journal of Sport \& Exercise Psychology, 16, S128-S157.

Turvey, M. T., Burton, G., Pagano, C. C., Solomon, H. Y., \& RuneSON, S. (1992). Role of the inertia tensor in perceiving object orientation by dynamic touch. Journal of Experimental Psychology: Human Perception \& Performance, 18, 714-727.

Turvey, M. T., \& Carello, C. (1995). Dynamic touch. In W. Epstein \& S. Rogers (Eds.), Handbook of perception and cognition: Vol. 5. Perception of space and motion (pp. 401-490). New York: Academic Press.

Turvey, M. T., Carello, C., Fitzpatrick, P., Pagano, C. C., \& KADAR, E. (1996). Spinors and selective dynamic touch. Journal of Experimental Psychology: Human Perception \& Performance, 22, $1113-1126$.

\section{NOTES}

1. For example, even extensive reviews of the literature on kinesthesis (e.g., Clark \& Horch, 1986; McCloskey, 1978; see also Shepherd, 1988) focus almost exclusively on the perception of the body. They may 
briefly address the perception of object weight, but only with respect to the effect of fatigue. Typically no mention is made regarding the perception of spatial properties of objects and surfaces by kinesthesis.

2. It is possible, however, that mechanical parameters other than $I_{i j}$ may come into play when an object is held as still as possible when conditions of minimal movement are imposed (see Burton \& Turvey, 1990; Carello, Fitzpatrick, Domaniewicz, Chan, \& Turvey, 1992; Lederman, Ganeshan, \& Ellis, 1996).

3. A useful summary is that $I_{i j}$ provides the domains for two sets of functions, one consisting of the principal moments of inertia or eigenvalues, that map onto perceived object "magnitudes," such as length, shape, and weight, and one consisting of the principal axes of inertia, or eigenvectors, that map onto perceived object or limb "directions." The one-to-one relation between $I_{i j}$ expressed as moments and products of inertia and $I_{i j}$ expressed as eigenvalues and eigenvectors (as well as other expressions of $I_{i j}$, such as the ellipsoid of inertia) has been discussed elsewhere (e.g., Fitzpatrick et al., 1994; Pagano \& Turvey, 1992, 1993; Turvey et al., 1992; see also Moon \& Spencer, 1986). For simplicity of exposition, the present discussion will focus on moments and products of inertia.

4. As discussed above, previous research reported that perceived partial length was a function of $I_{x x}$ calculated for only that part (e.g., Solomon et al., 1989b). A multiple regression of partial rod $I_{x x}$ calculated for the objects in Table 1 on the components of $I_{i j}$ reveals that $I_{x x}$ and $I_{y z}$ account for $97 \%$ of the variance, with the contribution of $I_{y z}$ dominant (partial $F$ s of 164.3 and 384.9 for $I_{x x}$ and $I_{y z}$, respectively). Considering that the tissue deformation consequences of wielding could only be the result of the entire rod, it appears likely that observers in these previous experiments were in fact sensitive to the components of $I_{i j}$ for the rod taken in its entirety, rather than $I_{x x}$ for only part of the object. Similarly, it has been reported that a wielded rod's gravitational torque, $N_{g}$ (sometimes referred to as "static" torque $N_{s}$ ), calculated as though the rod were being held stationary in a horizontal position, may account for $79 \%$ of the variance in perceived partial rod length (Chan, 1994). Since $N_{g}$ calculated in this manner covaries perfectly with $I_{y z}$, and the components of $I_{i j}$ taken together account for $97 \%$ of the variance in actual partial length, it is likely that the observers in Chan's experiment were in fact sensitive to $I_{i j}$ taken in its entirety rather than to $N_{\mathrm{g}}$ (which does not remain invariant during wielding). That is, one or more moments of inertia, along with $I_{y z}$, should account for the variance in perceived partial length not accounted for by $N_{g}$ in the regressions calculated by Chan. In fact, using the values presented in Chan's Table 3, the $N_{g}$ (which covaries perfectly with $I_{y z}$ ) and moment of inertia values account for $94 \%$ of the variance in perceived partial length when taken together in a multiple regression. More complete discussion of Chan (1994) can be found elsewhere (Carello, Santana, \& Burton, 1996; Turvey \& Carello, 1995).

5. Using the data of the individual subjects $(n=90), r^{2}=.72$, with only $I_{y z}$ significant after backwards elimination.

6. It was later discovered that in the two-added-mass condition, the masses placed above and below the hand were 49.0 and $49.6 \mathrm{~g}$, respectively. Thus the $I_{x x}$ and $I_{z z}$ values for this condition differ slightly.

(Manuscript received April 21, 1995; revision accepted for publication January 29, 1996.) 